\title{
WEAKLY CONTINUOUS ACCRETIVE OPERATORS
}

\author{
BY W. E. FITZGIBBON \\ Communicated by Fred Brauer, September 28, 1972
}

We shall be concerned with the autonomous differential equation

$$
u^{\prime}(t)+A u(t)=0, \quad u(0)=x,
$$

where $A$ is a weakly continuous possibly nonlinear operator mapping a reflexive Banach space $X$ to itself. Recently S. Chow and J. D. Schuur [2] have considered existence theory for ordinary differential equations involving weakly continuous operators on separable, reflexive Banach spaces.

We now make clear our notion of strong solutions to (1.1).

Definition 1.2. A function $u:[0, T) \rightarrow X$ is said to be a strong solution to the Cauchy problem

$$
u^{\prime}(t)+A u(t)=0, \quad u(0)=x,
$$

provided that $u$ is Lipschitz continuous on each compact subset of $[0, T)$, $u(0)=x, \quad u$ is strongly differentiable almost everywhere and $u^{\prime}(t)+A u(t)=0$ for a.e. $t \in[0, T)$.

By employing a variant of the Peano method we provide local solution to $(1.1)$.

LemMA 1.3. Let $X$ be a reflexive Banach space and suppose that $A$ is a weakly continuous operator with $D(A)=X$. Then there is a finite interval $[0, T)$ such that the Cauchy problem (1.1) has a strong solution on $[0, T)$.

Definition 1.4. An operator $A$ is said to be accretive provided that $\|x+\lambda A x-(y+\lambda A y)\| \geqq\|x-y\|$ for all $\lambda \geqq 0$ and $x, y \in D(A)$. T. Kato [5] has shown that this definition is equivalent to the statement that $\operatorname{Re}(A x-A y, f) \geqq 0$ for some $f \in F(x-y)$ where $F$ is the duality map from $X$ to $X^{*}$.

If we require that the operator $A$ be accretive we are able to extend the local solution of Lemma 1.3 to a global solution.

THEOREM 1.5. Let $X$ be a reflexive Banach space and suppose that $A$ is a weakly continuous accretive operator with $D(A)=X$. Then the Cauchy problem (1.1) has a unique strong global solution on $[0, \infty)$.

AMS (MOS) subject classifications (1970). Primary 47H15, 34H05; Secondary 47B44, 47D05.

Key words and phrases. Accretive, weakly continuous, semigroup of nonexpansive nonlinear operators. 
If we set $u(t)=T(t) x$ we obtain a semigroup of nonlinear nonexpansive operators $\{T(t): t \geqq 0\}$ which map $X$ to $X$. We can say that $\{T(t): t \geqq 0\}$ is the semigroup associated with $A$. The next theorem provides an exponential representation for $\{T(t): t \geqq 0\}$.

THEOREM 1.6. Let $A$ and $X$ satisfy the conditions of Theorem 1.5. Then the operator $A$ is $m$-accretive, i.e., $R(I+\lambda A)=X$ for all $\lambda \geqq 0$. If $\{T(t): t \geqq 0\}$ is a semigroup associated with $A$ then $T(t)$ may be represented as the pointwise limit

$$
T(t) x=\lim _{n \rightarrow \infty}(I+t / n A)^{-n} x .
$$

Moreover, for each fixed $t_{0}>0$, the operator $T\left(t_{0}\right)$ is weakly continuous.

The $m$-accretiveness of $A$ is obtained by considering the equation $u^{\prime}(t)+A^{\prime} u(t)=0$ where $A^{\prime}=A+I$. Once the $m$-accretiveness of $A$ has been established the exponential representation of $\{T(t): t \geqq 0\}$ follows immediately from a theorem of M. Crandall and T. Liggett [1]. The fact that $T\left(t_{0}\right)$ is weakly continuous is obtained by showing that $(I+\lambda A)^{-1}$ is weakly continuous for all $\lambda \geqq 0$ and employing estimates of Crandall and Liggett. The foregoing results may be applied to the rest point theory developed by C. Yen [10].

\section{REFERENCES}

1. M. Crandall and T. Liggett, Generation of semigroups of nonlinear transformations on general Banach spaces, Amer. J. Math. 93 (1971), 265-298.

2. S. Chow and J. D. Schuur, An existence theorem for ordinary differential equations in Banach spaces, Bull. Amer. Math. Soc. 77 (1971), 1018-1020.

3. E. Hille and R. S. Phillips, Functional analysis and semi-groups, rev. ed., Amer. Math. Soc. Colloq. Publ., vol. 31, Amer. Math. Soc., Providence, R.I., 1957. MR 19, 664.

4. T. Kato, Accretive operators and nonlinear evolution equations in Banach spaces, Proc. Sympos. Pure Math., vol. 18, part 1, Amer. Math. Soc., Providence, R.I., 1970, pp. 138-161. MR 42 \# 6663.

5. Nonlinear semigroups and evolution equations, J. Math. Soc. Japan 19 (1967), 508-520. MR 37 \# 1820.

6. R. H. Martin, Jr., A global existence theorem for autonomous differential equations in a Banach space, Proc. Amer. Math. Soc. 26 (1970), 307-314. MR 41 \#8791.

7. J. W. Neuberger, An exponential formula for one-parameter semi-groups of nonlinear transformations, J. Math. Soc. Japan 18 (1966), 154-157. MR 34 \#622.

8. S. Ôharu, Note on the representation of semi-groups of non-linear operators, Proc. Japan Acad. 42 (1966), 1149-1154. MR 36 \#3167.

9. G. F. Webb, Continuous perturbations of linear accretive operators in Banach spaces, J. Functional Analysis (to appear).

10. C. Yen, The convergence, periodicity and rest point behaviour of orbits in nonlinear semigroups of contractions, Thesis, Vanderbilt University, Nashville, Tenn., 1971.

Department of Mathematics, University of Houston, Houston, Texas 77004 